\title{
Blended learning for doctoral training in the context of the COVID-19 pandemic
}

\section{Aprendizagem híbrida para a formação doutoral no contexto da pandemia da COVID-19}

\section{Aprendizaje combinado para la formación doctoral en el contexto de la pandemia del COVID-19}

\author{
Yessy Villavicencio Simón ${ }^{1}$ iD, Ivan Gabriel Grajales Melian² iD , Cícero da Silva ${ }^{1,3}$ iD \\ ${ }^{1}$ Universidad Federal de Tocantins, Araguaína, Tocantins, Brasil. \\ 2 Universidad de Oriente, Santiago de Cuba, Cuba. \\ ${ }^{3}$ Universidad Estadual Paulista Júlio de Mesquita Filho, São José do Rio Preto, São Paulo, Brasil.
}

Autor correspondiente:

Cícero da Silva

Email: cicolinas@yahoo.com.br

Cómo citar: Simón, Y. V., Melian, I. G. G., \& da Silva, C. (2022). Blended learning for doctoral training in the context of the COVID-19 pandemic. Revista Tempos e Espaços em Educação, 15(34), e16685.

http://dx.doi.org/10.20952/revtee.v15i34.16685

\begin{abstract}
Distance learning has been an option to mitigate the global spread of the highly contagious disease, named COVID-19 (coronavirus), and declared a Pandemic by the World Health Organization (WHO) in March 2020. Social confinement as a public health strategy to address the health crisis has led to the adoption of new teaching methods in the new social sphere. Higher education contexts have gradually incorporated the opportunities offered by information and communication technologies (ICT). The purpose of the article is to present some theoretical reflections on the inclusion of the Blended Learning (B-Learning) methodological model in higher education, considering the changes that have occurred in the current situation due to the pandemic. Based on the review of the specialized scientific literature in relation to the integration of ICT in educational environments, the relevance of the advantages of technological tools in blended learning in the current context of the COVID-19 pandemic is recognized, specifically for the doctoral training process.
\end{abstract}

Keywords: Blended learning. COVID-19 pandemic. Higher education. Information and communication technologies.

\section{RESUMO}

A educação a distância tem sido uma opção para mitigar a disseminação mundial da doença altamente contagiosa, denominada COVID-19 (coronavírus), e declarada pandemia pela Organização Mundial de Saúde (OMS) em março de 2020. O distanciamento social como estratégia 
de saúde pública para enfrentar a crise sanitária tem influenciado a adoção de novas modalidades de ensino no novo ambiente social. Os contextos de ensino superior têm incorporado gradativamente as oportunidades oferecidas pelas Tecnologias da Informação e Comunicação (TIC). O objetivo do ensaio é apresentar algumas reflexões teóricas sobre a inserção do modelo metodológico da aprendizagem híbrida (Blended Learning ou B-Learning) no ensino superior, considerando as mudanças ocorridas na situação atual em decorrência da pandemia. Com base na revisão de literatura científica especializada em relação à integração das TIC em contextos educacionais, é reconhecida a relevância das vantagens das ferramentas tecnológicas na aprendizagem híbrida no contexto atual da pandemia da COVID-19, especificamente para o processo de formação em nível de doutorado.

Palavras-chave: Aprendizagem híbrida. Educação superior. Pandemia da COVID-19. Tecnologias da informação e comunicação.

\section{RESUMEN}

La educación a distancia ha sido una opción para mitigar la propagación mundial de la enfermedad altamente contagiosa, denominada COVID-19 (coronavirus), y declarada Pandemia por la Organización Mundial de la Salud (OMS) en el mes de marzo de 2020. El confinamiento social como estrategia de salud pública para enfrentar la crisis sanitaria, ha incidido en la adopción de nuevas modalidades de enseñanza en el nuevo ámbito social. Los contextos de educación superior han incorporado paulatinamente, las oportunidades ofrecidas por las Tecnologías de la Información y Comunicación (TIC). El propósito del ensayo es presentar algunas reflexiones teóricas sobre la inclusión del modelo metodológico de Aprendizaje Combinado (Blended Learning o B-Learning) en la educación superior, considerando los cambios ocurridos ante la situación actual por la pandemia. A partir de la revisión de la literatura científica especializada en relación con la integración de las TIC en los ámbitos educativos, se reconoce la pertinencia de las ventajas de las herramientas tecnológicas en el aprendizaje combinado en el actual contexto de pandemia del COVID-19, específicamente, para el proceso de formación doctoral.

Palabras clave: Aprendizaje combinado. Educación superior. Pandemia del COVID-19. Tecnologías de la información y las comunicaciones.

\section{INTRODUCCIÓN}

El confinamiento social y la cuarentena como estrategias de salud pública con el objetivo de prevenir la propagación mundial de la enfermedad altamente contagiosa, denominada COVID-19 (Síndrome Respiratorio Agudo Severo causado por coronavirus 2 - SARS-Cov-2), y declarada pandemia por la Organización Mundial de la Salud (OMS) en el 11 del mes de marzo de 2020 (OPAS/OMS, 2020), ha incidido en la adopción de nuevas modalidades de enseñanza y aprendizaje sobre la base de las necesidades de las instituciones educativas. Así, diversas alternativas didácticas se han implementado como medidas de prevención del coronavirus, durante el actual panorama caracterizado por la modificación de modalidades académicas, interrupción de la escolarización, inestabilidad económica y suspensiones de clases.

Se han adoptado medidas como la introducción o ampliación de la educación a distancia, estimular a docentes y administradores escolares a emplear aplicaciones, difundir contenidos educativos a través de la televisión y otros medios, proveer plataformas en línea, así como utilizar campañas de sensibilización o estrategias de comunicación sobre educación a distancia, destinadas a familias y alumnos (Cotino, 2020). La educación a distancia es denominada como "aprendizaje en línea e instrucción mediada por la tecnología, como cualquier curso en el que los estudiantes se separan entre sí o en forma presencial de la facultad [...]." (Abreu, 2020, p. 3).

Resultan válidas las sugerencias que la Secretaría General de la Universidad Nacional Autónoma de México, presenta en el documento Recomendaciones para la transición a la docencia 
no presencial (abril del 2020), sobre la base de la vasta experiencia de la educación universitaria en las modalidades educativas abierta y a distancia, así como en literatura especializada sobre el tema. Consecuentemente, se plantea que es preciso generar un entorno que propicie el aprendizaje autónomo y la enseñanza de estrategias de autogestión del conocimiento, a partir del establecimiento de objetivos, la distribución de actividades y la organización del tiempo. Asimismo, las condiciones de acceso a la tecnología por los estudiantes, impactan en la posibilidad de realizar las actividades a distancia que requieren procesamiento de información y una buena calidad de conexión a Internet. En tal sentido, es imprescindible la educación digital como "un salvavidas en una situación como la pandemia para garantizar unos mínimos de los objetivos que garantiza el derecho a la educación" (Cotino, 2020).

Por ello, las implicaciones sociológicas por el impacto de la pandemia del COVID-19, ha presentado la necesidad de redireccionar las acciones formativas hacia el manejo de las posibilidades de aplicación derivadas de las Tecnologías de la Información y la Comunicación (TIC), basado en una visión integradora del modelo metodológico de Aprendizaje Combinado (Blended Learning o B-Learning), como un atenuante en la búsqueda de una debida normalidad a las actividades educativas, el cual propicie mejorar la calidad de la educación ante los desafíos que enfrentan no solo los docentes, sino también los alumnos en tiempos de crisis.

El escenario vigente como resultado de la crisis sanitaria ha exigido algunos ajustes en contextos de educación superior. Se considera que, en los estudiantes de programas de formación doctoral, la situación actual de la pandemia del COVID-19 supone un reto y al mismo tiempo, una prueba de resiliencia entendida como capacidad de adaptación al enfrentarse a circunstancias adversas, pues también son docentes. Por tanto, urge la necesidad de comprender la implicación del concepto de resiliencia en la educación superior como un cambio de mentalidad, ya que la educación posee el desafío actual de garantizar una educación de calidad en sus estudiantes (Álvarez, 2020).

En este nuevo marco formativo de la enseñanza ante el COVID-19, se retoma una de las aristas señaladas por Morin (Oliva, 2020), en relación con el primer saber de la propuesta de siete saberes necesarios para la educación del futuro. El primer saber afrontar las incertidumbres, defiende que lo incierto genera una perspectiva de inseguridad a la actividad educativa que provoca la inestabilidad. De este modo, los docentes pueden modificar el desarrollo de su acción formativa teniendo en cuenta distintas alternativas de solución a los problemas para afrontar lo inesperado y ser más resilientes ante los cambios, en tanto forma de aprendizaje de las crisis.

La finalidad del trabajo es ofrecer algunas reflexiones sobre la importancia del aprendizaje combinado o blended learning y, en particular, la incidencia de las TIC en la adopción de esta modalidad de enseñanza, para el desarrollo de la formación posgraduada en un entorno educativo que presenta una acusada dificultad a partir de la crisis generada por el coronavirus, con énfasis en el nivel académico de doctorado. Es necesario lograr transformaciones en la educación superior que posibiliten fomentar el proceso de enseñanza y aprendizaje ante esta nueva realidad social, la cual precisa de adaptaciones a partir del aprovechamiento de las tecnologías de la información y la comunicación por los alumnos, y los docentes, quienes determinan la calidad del proceso educativo.

\section{CONSIDERACIONES TEÓRICAS SOBRE EL APRENDIZAJE COMBINADO EN CONTEXTO EDUCATIVO}

Teniendo en cuenta el método hermenéutico en la interpretación de la información obtenida de la revisión bibliográfica, el presente ensayo se sustenta en las investigaciones actuales sobre las implicaciones de un cambio en la educación superior como parte del sistema educativo, para enfrentar la pandemia del COVID-19 (Abreu, 2020; Álvarez, 2020; Costa \& Sousa, 2020; Cotino, 2020; lisue, 2020; Martins, 2020; Oliva, 2020). Se precisan las conceptualizaciones del término aprendizaje combinado o blended learning (Contreras, Penalba \& Eguia, 2006; Morales \& Ferreira, 2008; Esparaza, Salinas \& Glasserman, 2015; Juca, Carrión \& Juca, 2020), y la importancia de 
potenciar la utilización de las TIC como herramientas para crear ambientes de aprendizaje virtual (González, Perdomo \& Pascuas, 2017; Gargallo, 2018; Fernández et al., 2019; Moro, Dupotey \& Salgado, 2019), en relación con las potencialidades de su aplicación en esta modalidad educativa para asegurar un óptimo proceso educativo en tiempos de crisis, que responda a las exigencias de la universidad.

La experiencia de asumir la docencia virtual debido a la suspensión de clases ante el coronavirus, ha generado importantes cambios para transitar las actividades educativas de la modalidad presencial a la no presencial ante un nuevo contexto social. De esta manera, se han implementado programas de educación a distancia, la utilización de plataformas educativas abiertas con la intención del acceso remoto por los alumnos y profesores, así como el aprovechamiento de las tecnologías móviles de bajo costo con fines de enseñanza y aprendizaje para mitigar las interrupciones educativas. Sin embargo, se reconoce que los sistemas educativos no han logrado sacar el máximo provecho al recurso tecnológico, desde la comprensión que las TIC son herramientas transformadoras que facilitan afrontar las crisis educativas que afectan la presencialidad del estudiante en el aula (Oliva, 2020).

Los aspectos vinculados con la capacitación, el conocimiento y las creencias de los docentes sobre el amplio alcance de las Tecnologías de la Información y Comunicación, las cuales dominan la actual sociedad del conocimiento y su relación con las prácticas docentes, se han identificado entre las principales barreras para la integración de las tecnologías en la educación. De ahí la necesidad de la sensibilización y formación de los docentes para que su empleo no se circunscriba a su función informativa. Deben atenderse cuestiones como la definición del "modelo pedagógico a partir del cual identificar las necesidades y las tecnologías más apropiadas"; la existencia de una estrategia institucional que provea un adecuado desarrollo de las TIC en la enseñanza; así como "la promoción de entornos interactivos y colaborativos que favorezcan los procesos de enseñanza-aprendizaje" (Gargallo, 2018, p. 330).

En la misma línea discursiva, Fernández et al. (2019) justifican el aprovechamiento didáctico de las herramientas tecnológicas por el profesorado, para potenciar el proceso de aprendizaje con un carácter activo, dinámico, creativo, colaborativo y reflexivo desde una perspectiva de innovación educativa, acorde con el nuevo planteamiento educativo bajo el paradigma constructivista y conectivista de aprendizaje en red. Por ende, urge una adecuada capacitación del docente tanto en formación instrumental en lo que respecta a las TIC, como en lo relativo a la dimensión de las actitudes hacia estas. Ello posibilita plantear nuevas experiencias de aprendizaje que se centren en los estudiantes como protagonistas de los procesos de enseñanza-aprendizaje y favorezcan la implementación de la cultura digital en el proceso educativo, con lo cual se suscitan las denominadas pedagogías emergentes (Adell \& Castañeda, 2012, citados por Fernández et al., 2019).

Las tendencias en la aplicación de las TIC para la docencia universitaria coinciden en señalar un conjunto de indicaciones como:

avanzar hacia infraestructuras tecnológicas flexible y sostenible; fomentar la cultura digital a todos los niveles; diseñar el modelo pedagógico antes que el modelo tecnológico; gestionar un almacenamiento en constante crecimiento, hacia un modelo basado en la nube (cloud computing); utilizar modelos y arquitecturas abiertas que faciliten la adopción de nuevas plataformas de m-learning y herramientas sociales; fomentar el diálogo en red y los grupos de trabajo de docentes, una tendencia que debe estar encaminada hacia la creación de comunidades de aprendizaje; garantizar una gestión eficiente del cambio a través de la programación en las universidades de la innovación; proporcionar una actitud positiva y constructiva frente al cambio para evitar la brecha digital dentro de la propia universidad, facilitando la transición a nuevos modelos de aprendizaje; potenciar la creación de materiales docentes en abierto (bajo licencias creative commons) como alternativa a la proliferación de materiales en la red; y proporcionar al estudiante formación para las nuevas competencias que 
deben ser adquiridas para estas modalidades de aprendizaje basadas en TIC (Llorens, 2012; Pardo, Camarillo \& Jiménez, 2016, citados por Gargallo, 2018, p. 334-335).

Debido a que la acelerada integración de las herramientas virtuales, se ha convertido en una exigencia permanente en la mayoría de los nuevos contextos educativos durante la pandemia del COVID-19, es importante reflexionar sobre la importancia de capacitar al docente en la implementación didáctica de las tecnologías para cumplir las demandas de la educación. En este sentido, se considera que la utilización de las TIC en los momentos actuales, para la formación del alumno desde una participación activa como usuarios de las tecnologías, depende también del contexto de aprendizaje, los recursos disponibles, la orientación y las posibilidades de acceso a lo tecnológico.

Igualmente, los diversos "factores sociales, culturales y laborales influenciados por las tecnologías se combinan en un entorno centrado en el conocimiento, en el cual los procesos de enseñanza y aprendizaje exigen respuestas a las instituciones educativas que rebasan el modelo de actividades presenciales en el aula" (Bartolomé, 2004, citado por Esparaza, Salinas \& Glasserman, 2015, p. 3). De ahí surgen algunas modalidades de enseñanza que incorporan tecnologías, como el aprendizaje combinado (blended learning o b-learning), el cual adopta el modelo e-learning más conocido como el aprendizaje electrónico, basado en la educación a distancia por medio de la incorporación de las herramientas TIC, y la docencia presencial; "es aplicable a diferentes áreas del conocimiento y permite definir entornos virtuales accesibles con esquemas innovadores" (González, Perdomo \& Pascuas, 2017, p. 151).

Estudios realizados coinciden en señalar que la inclusión del modelo metodológico de aprendizaje combinado, resulta favorable en tanto "es una modalidad semipresencial de estudios, que combina los componentes de la enseñanza virtual (aulas virtuales, herramientas informáticas, Internet) y diversos métodos como prácticas de gestión del conocimiento, incluyendo salas presenciales (cara a cara) y el aprendizaje a ritmo individual". Por ende, se define como la integración de la formación no presencial y la formación presencial, con la posibilidad de disponer de un profesor como tutor de los cursos (Coaten, 2003; Marsh et al., 2003, citados por Contreras, Penalba \& Eguia, 2006; Morales \& Ferreira, 2008).

Por ello, también el término se delimita como modelo mixto, mezclado, híbrido y virtualpresencial, pues el empleo de las TIC aporta ventajas que podrían incorporarse a entornos de modalidad presencial, con lo cual se obtiene una combinación ideal entre actividades presenciales y no presenciales. Por un lado, esta modalidad rescata la flexibilidad de tiempo y espacio del ambiente virtual, así como "la igualdad de oportunidad de participación, la variedad de materiales de apoyo, el seguimiento personalizado y la autogestión" del conocimiento (Esparaza, Salinas \& Glasserman, 2015, p. 3).

Para cumplir los objetivos y el porcentaje de las sesiones dedicadas a la presencialidad, se deben establecer los elementos adecuados de la educación a distancia, la metodología para el uso de los materiales, y analizar el software o la plataforma virtual para envasar actividades. Asimismo, los profesores deben prestar atención a los objetivos, las actividades y su distribución en el módulo presencial, el cual posibilita la retroalimentación (feedback) para la internalización de los conocimientos adquiridos, pues las sesiones cara a cara se usan para reforzar los contenidos programados que la educación a distancia como forma de enseñanza remota y mayoritariamente asíncrona, o la no-presencialidad pudiera no cubrir totalmente (Morales \& Ferreira, 2008).

Las investigaciones desarrolladas demuestran que la efectividad de un modelo semipresencial para la enseñanza-aprendizaje, depende de la planificación a partir de un análisis de las necesidades e intereses de los estudiantes, basado en los principios metodológicos en que se sustenta el enfoque, diseño e implementación apoyada por los recursos tecnológicos apropiados que puedan servir de apoyo al proceso educativo. En esta misma línea, se asume que la 
incorporación de este modelo al proceso de formación doctoral resulta una opción para la educación superior ante el escenario vigente en el contexto cubano (Morales \& Ferreira, 2008).

En consonancia con el actual entramado tecnológico, social y comunicativo de las universidades como pilares tecnosociales para "la expansión y difusión del conocimiento global, el empoderamiento de la ciudadanía, la innovación educativa, la transferencia del conocimiento y dinamizadora del desarrollo profesional y la cohesión social e integradora en el tejido tecnológico y económico de la sociedad del conocimiento para el desarrollo y el progreso humano" (Montoya, Verdezoto \& Cornejo, 2019, p. 3-4), se postula que estas deben adaptar los procesos de formación atendiendo a las características y necesidades actuales de los estudiantes (Fernández et al., 2019). Como bien afirma Loidi (2019), la educación superior constituye un eje temático de la reciente Conferencia Regional de Educación Superior (Cres, 2018), y un aspecto central del Objetivo de Desarrollo Sostenible Educación de Calidad (ODS) de la Organización de las Naciones Unidas (ONU, 2015), con gran incidencia sobre los restantes siete ejes temáticos y diecisiete ODS. La calidad y pertinencia en la educación superior, ha sido un tema recurrente a nivel regional desde la Conferencia Regional de Educación Superior para América Latina y el Caribe en La Habana en 1996 y objeto de seguimientos en los congresos internacionales UNIVERSIDAD que ya van por la oncena edición, y alternan con los congresos de PEDAGOGÍA ya en su 16.a edición.

La formación doctoral como actividad científica en Cuba, cuenta con reconocidos programas de doctorado, entre ellos en ciencias pedagógicas, los cuales responden a las demandas y necesidades del personal docente para elevar el desarrollo profesional en el sistema educativo con rigor científico y calidad, a partir de la interrelación entre la superación y la investigación. El perfeccionamiento de la calidad y la eficiencia del posgrado debe expresarse en la ejecución de actividades de formación continua en función del empleo creador de las tecnologías de la información y las comunicaciones (Loidi, 2019).

De acuerdo con Álvarez (2020, p. 410), "se demanda de los doctorandos una mayor cantidad de competencias relacionadas con la tecnología que impliquen a su vez elementos como: creatividad, pensamientos, negociación, inteligencia emocional y resiliencia". Por tanto, es imprescindible el análisis del tema de la educación virtual dentro de las políticas de estado de un país y de los que toman decisiones en el ámbito de la educación nacional.

En un marco de aprendizaje combinado en las universidades cubanas en tiempos de la pandemia actual del COVID-19, los recursos tecnológicos constituyen una gran ventaja como herramientas didácticas, en tanto "permiten el acceso y procesamiento de la información, la gestión de conocimientos, una adecuada conexión a una red y facilitan el proceso de enseñanzaaprendizaje" (Moro, Dupotey \& Salgado, 2019, p. 155). Conforme estos autores, las oportunidades de acceso al conocimiento que ofrecen las TIC como fuentes de iniciativas para responder a los retos que el mundo de la tecnología impone actualmente a la educación superior, conduce a la necesidad de generalizar su empleo para favorecer el desarrollo del proceso formativo del profesional con calidad mediante las técnicas adecuadas. Se trata de que los profesores de las disciplinas planifiquen materiales adecuados para los doctorandos, con el fin de distribuir, ampliar, generar conocimientos, profundizar en los contenidos planteados, debatir, investigar y elaborar la información en el ámbito formativo.

Se asume el criterio de Fernández et al. (2019) acerca de que las tecnologías digitales pueden mejorar los procesos de enseñanza a través de la innovación en materiales didácticos y metodologías didácticas más activas y eficaces. Además, entre las ventajas de su implementación en el ámbito universitario, se manifiesta la posibilidad de facilitar el diálogo y la investigación educativa, como recursos educativos en los procesos de tutorización y el seguimiento didáctico. Cabe señalar que el docente debe realizar una adecuada planificación y diseño de un proyecto pedagógico sólido a partir de la situación específica de cada aula. Por tanto, el manejo de las TIC debe alternarse con el uso de otros recursos de enseñanza y aprendizaje de una forma 
complementaria.

Según Gargallo (2018), resulta imprescindible la presencia de conceptos como la flexibilidad, la innovación, la adaptabilidad o la interconectividad desde el punto de vista organizativo y estratégico en un modelo educativo centrado en el papel activo del estudiante y encaminado al desarrollo de competencias en un contexto colaborativo e interactivo, que refuerce el aprendizaje autónomo y garantice alcanzar resultados superiores. Así, resulta más apropiado hablar de entornos educativos tecnológicamente enriquecidos (Llorens, 2012, citado por Gargallo, 2018).

El aprendizaje combinado posibilita la aplicabilidad de las herramientas tecnológicas en los programas de formación doctoral, al propiciar la utilización de materiales escritos en soporte electrónico como libros electrónicos (e-book) o lecturas en archivo PDF, la interacción vía correo electrónico con los estudiantes y profesor, las redes sociales como contextos de relación, los repositorios de contenido como espacios de colaboración, la búsqueda de información en Internet y el uso de entornos virtuales de aprendizaje (EVA), con la intención de lograr los objetivos del proceso. Además, aprovecha del ambiente presencial, la interacción cara a cara, la espontaneidad, la resolución inmediata de dudas y el acceso directo al profesor, lo que da lugar al modelo ecléctico o integrado. Así, la explotación de los recursos tecnológicos de manera flexible, contribuye a garantizar la interacción estudiantes-profesor y estudiantes-estudiantes, a partir del uso de los distintos recursos computacionales como el chat o foro, las multimedias y apps, facilitan intercambiar lo aprendido de modo individual en la sala de clase, enriquecer el aprendizaje y lograr la consolidación de los contenidos (Esparaza, Salinas \& Glasserman, 2015).

De este modo, constituye una modalidad educativa emergente y ajustable, o sea, un sistema de adaptación que contribuye a articular las necesidades, orientar el desarrollo de habilidades cognitivas y el pensamiento crítico y constructivo, vinculados a la toma de decisiones por medio de la observación, participación y reflexión. En otras palabras, el blended learning promueve el aprendizaje autónomo y colaborativo, que implica la interactividad entre el tutor y estudiante en cuanto el cumplimiento de tareas durante el curso (González, Perdomo \& Pascuas, 2017).

Si bien la integración progresiva de las herramientas tecnológicas es un aspecto positivo, también surge la siguiente dificultad en la enseñanza universitaria cubana: "la problemática de la capacidad en cuanto a cantidad de computadoras en buen estado, computadoras con buen rendimiento y capacidad suficiente para soportar software" (Moro, Dupotey \& Salgado, 2019, p. 155). En este orden, la situación requiere la necesidad y relevancia de un análisis profundo y constante por los equipos directivos de los ámbitos educativos, que posibilite ajustes pertinentes en los momentos actuales, de modo que sea óptimo el aprovechamiento de las potencialidades que ofrecen las redes en la implementación del modelo metodológico de aprendizaje combinado en la educación superior cubana y latinoamericana en general.

\section{CONSIDERACIONES FINALES}

La educación superior debe adaptarse a las crisis originadas por la pandemia del COVID-19, en particular, el proceso de formación de doctorandos desde la adopción de la modalidad de aprendizaje combinado (blended learning o b-learning), la cual integra sesiones presenciales y virtuales. Este modelo semipresencial está vinculado a la aplicabilidad de las TIC y es ajustable a las necesidades educativas, de ahí que propicia favorecer la formación doctoral del profesor teniendo en cuenta la articulación de diferentes técnicas y herramientas tecnológicas para el desarrollo de actividades tanto presenciales como virtuales.

El análisis de la literatura científica especializada en relación con el ámbito del aprendizaje combinado permite reconocer las potencialidades del uso de las TIC en esta metodología, en tanto favorece un proceso interactivo y efectivo en el contexto universitario al mejorar la apropiación de conocimientos, la interacción y generar a su vez, la calidad del proceso de formación doctoral en los contextos educativos. La aplicabilidad de las herramientas tecnológicas en la realidad educativa 
exige la capacitación de doctorandos y docentes para una adecuada apropiación de su manejo, en aras de adaptar el proceso de enseñanza-aprendizaje a los tiempos de pandemia.

En síntesis, permite la flexibilidad horaria, accesibilidad a la información, rapidez en la comunicación, desarrollo y actualización de contenidos, y la solución de problemas de la cotidianidad como desafío en un nuevo entorno educativo. Puede plantearse que el actual contexto de la pandemia del COVID-19 implica una actitud flexible y abierta en los estudiantes de programas de formación doctoral, quienes también ejercen la docencia en un escenario complejo. Por consiguiente, se precisa de la integración de las TIC en la educación superior, como núcleo de transformación de los procesos formativos que requieren de la generación y actualización del conocimiento desde la modalidad educativa de aprendizaje combinado o blended learning.

Contribuciones de los autores: Simón, Y. V.: concepción y diseño, adquisición de datos, análisis e interpretación de datos, redacción del artículo, revisión crítica de contenido intelectual importante; Melian, I. G. G.: concepción y diseño, adquisición de datos, análisis e interpretación de datos, redacción del artículo, revisión crítica de contenido intelectual importante; da Silva, C.: concepción y diseño, adquisición de datos, análisis e interpretación de datos, redacción del artículo, revisión crítica de contenido intelectual importante. Todos los autores han leído y aprobado la versión final del manuscrito.

Aprobación de ética: No aplica.

Agradecimientos: Los autores agradecen el financiamiento para este estudio proporcionado por CAPES (Coordenação de Aperfeiçoamento de Pessoal de Nível Superior - Brasil), Código Financiero 001, y CNPq (Conselho Nacional de Desenvolvimento Científico e Tecnológico - Brasil), Código Financiero 164941/2020-7.

\section{REFERENCIAS}

Abreu, J. L. (2020). Tiempos de Coronavirus: La Educación en Línea como Respuesta a la Crisis. Daena: International Journal of Good Conscience, 15(1), 1-15. http://www.spentamexico.org/v15-n1/A1.15(1)1-15.pdf

Álvarez De Barrios, A. M. (2020). Doctorandos en resiliencia ante la pandemia y las nuevas alternativas de aprendizaje. Universidad y Sociedad, 12(4), 407-410. https://rus.ucf.edu.cu/index.php/rus/article/view/1662/1667

Codeic Secretaría General Universidad Nacional Autónoma de México. (2020). Recomendaciones para la transición a la docencia no presencial. México: UNAM. https://www.codeic.unam.mx/index.php/recomendaciones-para-la-transiciona-la-docencia-no-presencial/

Contreras Espinosa, R., Penalba, F. A., \& Eguia Gómez, J. L. (2006). Tendencias en la educación: aprendizaje combinado. Theoria, 15(1), 111-117. https://www.redalyc.org/pdf/299/29915111.pdf

Costa, M. R. M., \& Sousa, J. C. (2020). Educação a Distância e Universidade Aberta do Brasil: reflexões e possibilidades para o futuro pós-pandemia. Thema, 18, 124-135. https://doi.org/10.15536/thema.V18.Especial.2020.124-135.1832

Cotino Hueso, L. (2020). La enseñanza digital en serio y el derecho a la educación en tiempos del coronavirus. Revista de Educación y Derecho, 21, p. 1-29. https://doi.org/10.1344/REYD2020.21.31283

Esparaza Moguel, M. C., Salinas Urbina, V, \& Glasserman Morales, L D. (2015). La gestión del aprendizaje en la modalidad b-learning frente a la modalidad presencial en la enseñanza de la gramática inglesa. Apertura, 7(2), 1-10. http://www.redalyc.org/articulo.oa?id=68842702001

Fernández Márquez, E. et al. (2019). La competencia digital en la docencia universitaria. Barcelona: Ed. OCTAEDRO, S.L.

Gargallo Castel, A. F. (2018). La integración de las TIC en los procesos educativos y organizativos. Educar em Revista, 34(69), 325-339. https://www.scielo.br/pdf/er/v34n69/0104-4060-er-34-69-325.pdf

González Aldana, M. A., Perdomo Osorio, K. V., \& Pascuas Rengifo, Y. (2017). Aplicación de las TIC en modelos educativos blended learning: una revisión sistemática de literatura. Sophia, 13(1), 144-154.

http://www.redalyc.org/articulo.oa?id=413750022015

IISUE. (2020). Educación y pandemia. Una visión académica. México: UNAM.

http://www.iisue.unam.mx/nosotros/covid/educacion-y-pandemia

Juca Maldonado, F., Carrión González, J., \& Juca Abril, A. (2020). B-Learning y Moodle como estrategia en la educación universitaria. Conrado, 16(76), 215-220. https://conrado.ucf.edu.cu/index.php/conrado/article/view/1476/1461 
Loidi, J. R. S. (2019). Universidad y sistema educativo. Articulación, calidad y pertinencia para el desarrollo. Islas, 61(193), 143-159. http://islas.uclv.edu.cu/index.php/islas/article/view/1172/932

Martins, R. X. (2020). A COVID-19 e o fim da educação a distância: um ensaio. Revista De Educação a Distância, 7(2), 242-256. https://www.aunirede.org.br/revista/index.php/emrede/article/view/620

Montoya, Y. A. C., Verdezoto, V. H., \& Cornejo, S. A. G. (2019). Experiencias innovadoras y transformación socioeducativa aprendizaje ubicuo con estudiantes universitarios, aplicado en América Latina. In Memorias del quinto Congreso Internacional de Ciencias Pedagógicas de Ecuador: Aprendizaje en la sociedad del conocimiento - modelos, experiencias y propuestos (pp. 174-185). Instituto Superior Tecnológico Bolivariano.

Morales Ríos, S., \& Ferreira Cabrera, A. (2008). La efectividad de un modelo de aprendizaje combinado para la enseñanza del inglés como lengua extranjera: estudio empírico. Revista de Lingüística Teórica y Aplicada, 46(2), 95118. https://scielo.conicyt.cl/pdf/rla/v46n2/art06.pdf

Moro Ortiz, M. A., Dupotey Varela, M. N., \& Salgado Castillo, A. (2019). Las TIC como herramienta didáctica. un estudio en la Universidad de Oriente. Revista Electrónica Formación y Calidad Educativa (REFCaIE), 7(3), 151-160. https://refcale.uleam.edu.ec/index.php/refcale/article/download/2742/1854

Oliva, H. A. (2020). La Educación en tiempos de pandemias: visión desde la gestión de la educación superior. https://www.disruptiva.media/la-educacion-en-tiempos-de-pandemias-vision-desde-la-gestion-de-la-educacionsuperior/

Organização Pan-Americana de Saúde / Organização Mundial de Saúde - OPAS/OMS (2020). Histórico da pandemia de COVID-19. Folha informativa sobre COVID-19, OPAS. https://www.paho.org/pt/covid19/historico-da-pandemia-covid$\underline{19}$

Recibido: 31 de octubre de 2022 | Aceptado: 22 de diciembre de 2022 | Publicado: 14 de enero de 2022

This is an Open Access article distributed under the terms of the Creative Commons Attribution License, which permits unrestricted use, distribution, and reproduction in any medium, provided the original work is properly cited. 